\title{
Acute Pulmonary Artery Obstruction as the Primary Manifestation of a Rapidly Growing Intimal Sarcoma in a 54-Year-Old Patient
}

\author{
Sumi Westhofen ${ }^{1}$ Christian Kugler ${ }^{2}$ Hermann Reichenspurner ${ }^{1}$ Tobias Deuse ${ }^{1}$ \\ ${ }^{1}$ Department for Cardiovascular Surgery, University Heart Center \\ Hamburg, Hamburg, Germany \\ 2 Department for Thoracic Surgery, Lung Clinic Grosshansdorf, \\ Großhansdorf, Germany \\ Address for correspondence Sumi Westhofen, MD, Department for \\ Cardiovascular Surgery, University Heart Center Hamburg, \\ Martinistraße 52, Hamburg 20246, Germany \\ (e-mail: f.westhofen@uke.de).
}

Thorac Cardiovasc Surg Rep 2016;5:36-38.
Abstract
Keywords
- thoracic surgery
- bronchial disease
- chest wall
- histology
- lung cancer treatment
- lung cancer
- diagnosis

Pulmonary artery sarcoma is a rare malignant neoplasm that is often misdiagnosed and most often only recognized postmortem during the autopsy. We present the case of a male patient with a rapidly progressive pulmonary tumor who underwent urgent pneumonectomy for increasing symptoms of chest pain and septic clinical picture. Histological analysis revealed the diagnosis of a pulmonary artery sarcoma. Despite an R1-resection and adjuvant chemotherapy, the patient is in good clinical health and free of tumor relapse 1 year after the surgery.

\section{Introduction}

Pulmonary artery sarcoma was first described by Mandelstamm in $1923 .^{1}$ With an incidence ranging between 0.001 and $0.03 \%$, as described in the literature, it has only been reported in a few hundred cases up to now. It accounts for only 2 to $3 \%$ of all soft tissue sarcomas in adults, typically originates from the intimal layer of the vessel, and occurs most often in middle-aged patients. Early diagnosis and treatment are crucial for these patients, since this neoplasm shows a very aggressive growth pattern. Diagnosis though, is often difficult and delayed due to the unspecific and misleading symptoms. Frequently, patients are misdiagnosed with pulmonary thromboembolism and are erroneously treated with anticoagulation or thrombolysis for a long time. ${ }^{2}$ Even if treated early with surgery and adjuvant therapeutic strategies, mortality is higher than $80 \%$ after 12 months. $^{3-7}$ The patient we report on survived an urgent operation, which was necessary for worsening symptoms and an increasingly septic course. The rapidly growing tumor completely occluded the left main pulmonary artery and already protruded into the right ventricular outflow tract.

received

April 11, 2016

accepted after revision

June 14, 2016

published online

August 30, 2016

\section{Case Report}

We herein report on a 54-year-old male patient without a medical history, who presented with acute onset of nonexercise-related chest pain. A chest X-ray revealed suspicious solitary pulmonary nodules in a racemose cluster in the left upper pulmonary lobe. The subsequent computed tomography (CT) scan verified solitary soft tissue lesions extending from the left hilum to the lateral left upper lobe pleura with in-growth into adjacent blood vessels. In addition, an abdominal scan showed hepatic lesions consistent with cysts or cystic metastases. Blood tests showed no abnormalities. In a transbronchial biopsy, no malignant cells or pathogenic microorganisms were detected. The patient decided to discharge himself from the hospital. Within 24 hours he developed recurrent episodes of fever with a beginning systemic septic reaction. Systemic antibiotic therapy did not show an improvement of the clinical situation. Fever episodes occurred repeatedly in a cyclic manner. Blood test results showed a procalcitonin level of over $20 \mu \mathrm{g} / \mathrm{L}$. Blood culture results were negative. CT scans were then repeatedly
License terms Stuttgart · New York

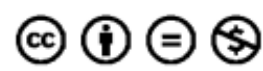




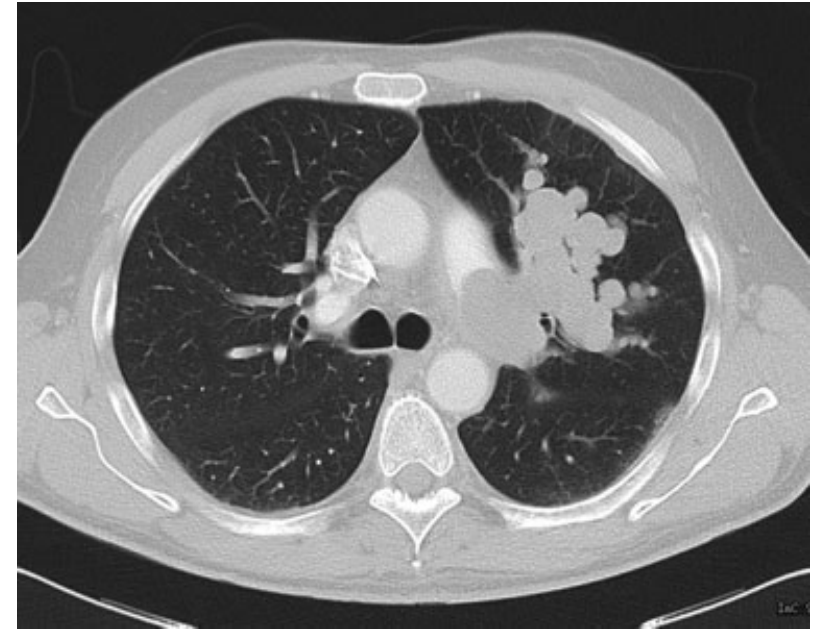

Fig. 1 CT scan preoperatively showing a rapid progress of the tumor mass, here it already shows the typical polypoid growth pattern, like a bunch of grapes. CT, computed tomography.

performed and revealed a rapid progress of the tumor mass (-Fig. 1). A complete occlusion of the left main pulmonary artery became apparent, with tumor protruding into the pulmonary trunk and the right ventricular outflow tract. A lung perfusion/ventilation scintigraphy showed a complete dysfunction of the left lung, probably causing the acute septic condition of the patient. Based on the progressive character of the disease, the decision for a left-sided pneumonectomy with extracorporeal circulatory support for pulmonary trunk repair was made. The operation was planned and realized in an interdisciplinary team approach of cardiac and thoracic surgeons.

The procedure was performed via a left anteroaxillary thoracotomy. The lung was found strongly attached to the chest wall. An extended pneumonectomy with partial pleurectomy and pericardiectomy due to tumor infiltration was performed, and included lymph node dissection. Palpable tumor nodes were soft. The left femoral vein and the descending aorta were cannulated and cardiopulmonary bypass was commenced. The pulmonary veins and the left main bronchus were stapled and transected. The left pulmonary artery was circularly excised from the pulmonary trunk and the tumor, which had already protruded into the right ventricular outflow tract was removed (-Fig. 2). The pulmonary trunk was repaired with bovine pericardium patch (Vascutek Deutschland GmbH, Hamburg, Germany). The pulmonary artery was filled with a mucinous, amber-colored mass. In an intraoperatively performed frozen section, the dignity of the explanted tumor could not be specified. Histological analysis and tumor typing revealed the diagnosis of a myxoid, spindle-cell intimal sarcoma FNCLCC (French Federation of Cancer Centers Sarcoma Group) grade III, with intravasal dissemination into the parenchyma of left upper and lower lobe (-Fig. 3). Further examination and analyses of the suspicious liver findings showed no indication for malignancy. No other systemic metastases were found. The patient recovered fully and quickly. Adjuvant chemotherapy with six

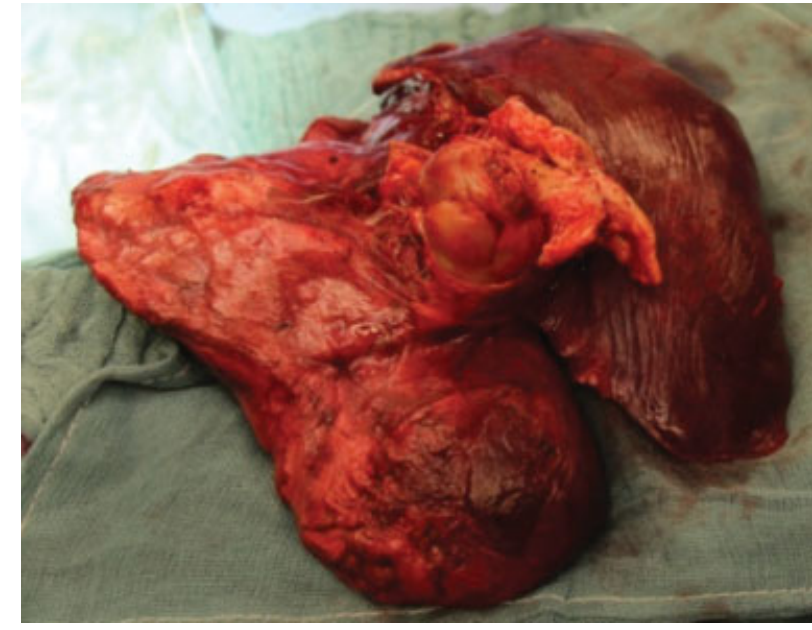

Fig. 2 Resected specimen showing the tumor mass infiltrating the pulmonary artery.

cycles of ifosfamide and etorubicin was initiated due to positive resection margin at the pulmonary artery (R1). After 1 year of follow-up, the patient is in a good clinical condition and tumor free, without signs of relapse. Under regular CT monitoring (every 6 months) he now receives a maintenance treatment with trofosfamide.

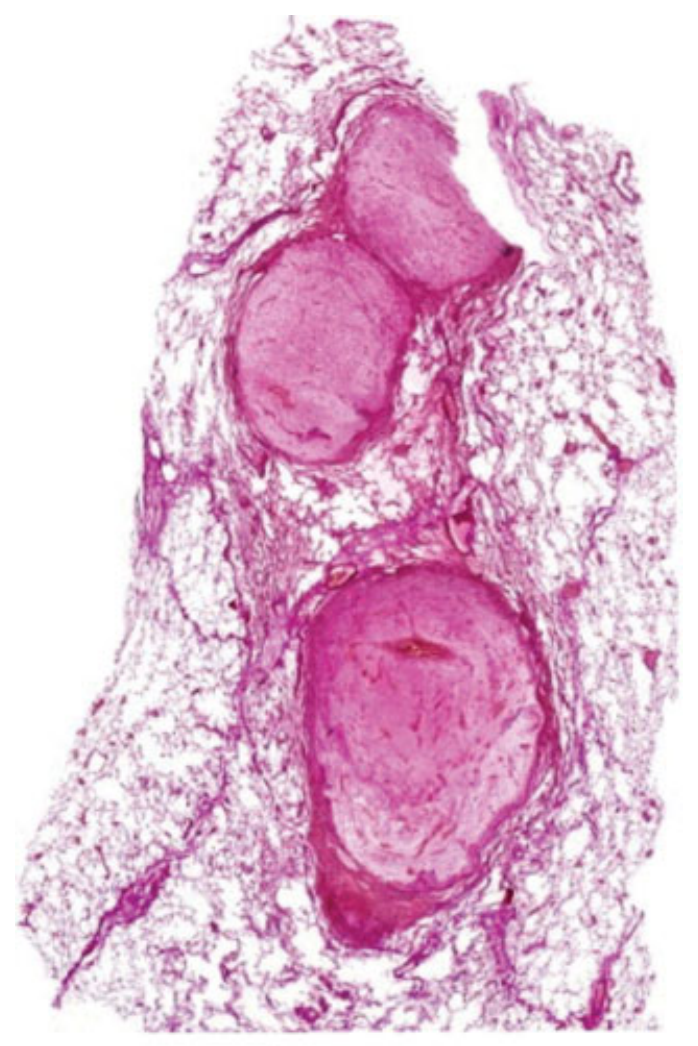

Fig. 3 Histological section of the myxoid tumor mass (EVG-stain, threefold enlargement), with canaliculary expansion inside the pulmonary vessels, spindle-cell formation with fibroelastic components. EVG, Elastic Van Gieson. 


\section{Discussion}

Pulmonary artery sarcomas are of mesenchymal cell origin, the etiology is still unknown. Epidemiological data suggest a peak at the age of 50 years without gender predominance. Extrathoracic metastases are less common, and typically affect skin, brain, lymph nodes, or kidneys. Median survival time of the pulmonary intimal sarcoma without therapy ranges around 1.5 months, and surgical resection is the treatment of choice. The value of adjuvant chemotherapy or radiotherapy is still unclear. ${ }^{1,8}$ Even if treated, long-term survival is still poor. Initial symptoms can be misleading, and pulmonary thromboembolism is an important differential diagnoses. So in many cases, the correct diagnosis comes too late for the patient. Diagnostic workup, however, should not postpone surgery, as this tumor is very aggressive and growing fast, and diagnostic tools other than histologic workup of the resected tumor mass are not reliable. Even transbronchial biopsies cannot confirm the diagnosis. In the presented case, the clinical picture of a septic episode complicated the diagnostic workup, as it suggested an infection rather than a malignant process. ${ }^{9,10}$ Nevertheless, an urgent operation was indicated and performed due to the rapid disease progress and the worsening clinical condition of the patient. Additionally, the proximity to the heart required an interdisciplinary team approach and operation under heart-lung machine protection to enable a nearly complete resection of the tumor. Even if prognosis improvement through adjuvant chemotherapy is not validated in the existing literature, our patient seems to have responded very well to it, so he is now tumor free and asymptomatic 1 year after surgery.

\section{Conclusion}

In patients with vascular-filling defects expanding the vessel lumen that are suspicious of pulmonary embolism, a pulmonary artery sarcoma should be considered as a differential diagnostic. Especially, if symptoms are not typical of pulmonary embolism or the patient does not improve with anticoagulation or lysis. Timely surgical resection with an adjuvant medical treatment at experienced centers may be ideal.

\section{References}

1 Mandelstamm M. Über primäre Neubildungen des Herzens. Virchows Arch Pathol Anat 1923;245:43-54

2 El-Sayed Ahmed MM, Aftab M, Al-Najjar RM, de la Cruz KI, Benjamin RS, Hallman CH. Pulmonary artery sarcoma mimicking pulmonary embolism. Tex Heart Inst J 2014;41(5):515-517

3 Grimer R, Judson I, Peake D, Seddon B. Guidelines for the management of soft tissue sarcomas. Sarcoma 2010;2010:506182

4 Nonomura A, Kurumaya H, Kono N, et al. Primary pulmonary artery sarcoma. Report of two autopsy cases studied by immunohistochemistry and electron microscopy, and review of 110 cases reported in the literature. Acta Pathol Jpn 1988;38(7):883-896

5 Blackmon SH, Rice DC, Correa AM, et al. Management of primary pulmonary artery sarcomas. Ann Thorac Surg 2009;87(3):977-984

6 Mussot S, Ghigna MR, Mercier O, et al. Retrospective institutional study of 31 patients treated for pulmonary artery sarcoma. Eur J Cardiothorac Surg 2013;43(4):787-793

7 Coli A, Parente P, Bigotti G. Pulmonary artery sarcoma: an insidious tumor still diagnosed too late. Analysis of the literature and report of a case. J Exp Clin Cancer Res 2007;26(1):151-156

8 Cox JE, Chiles C, Aquino SL, Savage P, Oaks T. Pulmonary artery sarcomas: a review of clinical and radiologic features. J Comput Assist Tomogr 1997;21(5):750-755

9 Wong HH, Gounaris I, McCormack A, et al. Presentation and management of pulmonary artery sarcoma. Clin Sarcoma Res 2015;5(1):3

10 Mellwig KP, Schmidt H, Gleichmann U, Raute-Kreinsen U. Metastasizing round-cell sarcoma of the right atrium [in German]. Dtsch Med Wochenschr 1991;116(40):1505-1508 